\begin{tabular}{|c|c|c|}
\hline \multicolumn{3}{|c|}{$\begin{array}{r}\text { Моделирование пищевых систем для алиментарной корр } \\
\text { соотношения полиненасыщенных жирных кислот } \\
\text { в организме человека }\end{array}$} \\
\hline Татьяна В. Алексеева & zyablova@mail.ru & (D) 0000-0001-8035-7293 \\
\hline Евгений О. Здоровцев & Zenya7777@mail.ru & (D) $0000-0002-5126-8723$ \\
\hline Людмила В. Малакова & mixbox3@mail.ru & (D) $0000-0003-0481-4175$ \\
\hline Анастасия П. Фурсова & nasti999@ gmail.com & (D) $0000-0003-0481-4290$ \\
\hline Александр Е. Столяров & stolyarov1997@bk.ru & (D) 0000-0003-3086-3280 \\
\hline Екатерина С. Талтынова & ekaterynageeva@mail.ru & (D) $0000-0001-6749-8227 x$ \\
\hline
\end{tabular}

1 Воронежский государственный университет инженерных технологий, пр-т Революции, 19, г. Воронеж, 394036, Россия

Аннотация. Положительное $\quad$ влияние на организм человека полиненасыщенных жирных кислот подтверждено многочисленными исследованиями. Поэтому представляется актуальным создание пищевых систем для обогащения продуктов массового потребления физиологически активными и эссенциальными веществами, в частности полиненасыщенными жирными кислотами. Эффект алиментарного шунтирования организма человека полиненасыщенными жирными кислотами зависит от множества факторов, самыми существенными считаются их количество в рационе, состав, соотношение полиненасыщенных и насыщенных жирных кислот, наличие антиоксидантов. Цель исследований - разработка методики управления качеством пищевых систем для балансирования соотношения $\omega-6$ и $\omega-3$ полиненасыщенных жирных кислот. Был разработан программный продукт, позволяющий подбирать соотношения компонентов в пищевой композиции для заданных соотношений $\omega-6 / \omega-3$. Для примера была выбрана пищевая обогащающая система, состоящая из жмыха зародышей пшеницы, масел амаранта и тыквы. Созданный программный продукт позволяет работать и с другими пищевыми системами. В результате обработки данных была получена номограмма для определения процентного соотношения компонентов $\omega-6 / \omega-3$ жирных кислот в пищевой системе. Разработан программный продукт, позволяющий подобрать в пищевых композициях по двум зафиксированным количественным значениям компонентов содержание третьего компонента, удовлетворяющего заданному соотношению $\omega-6 / \omega-3$ кислот. Предложен вариант пищевой композиции и проведена апробация работы разработанного программного продукта. Установлено, что пищевая обогащающая система на основе жмыха зародышей пшеницы, масел амаранта и тыквы может применяться для обогащения продуктов питания полиненасыщенными жирными кислотами, при этом создается возможность целенаправленного регулирования соотношения $\omega-6 / \omega-3$ жирных кислот. Разработанное программное обеспечение позволяет в условиях производства в зависимости от наличия определенных наименований сырьевых источников подбирать оптимальные варианты пищевых композиций и выбирать наиболее приемлемый в сложившейся производственной ситуации вариант, принимать решения по движению сырья на предприятии после анализа полученных данных.

Ключевые слова: полиненасыщенные жирные кислоты, баланс ПНЖК, омега-3, омега-6, оптимизация, программирование

\title{
Modeling of food systems for nutritional correction of the ratio of polyunsaturated fatty acids in people
}

\begin{tabular}{llll}
\hline \hline Tatyana V. Alekseeva & 1 & zyablova@mail.ru & (1) 0000-0001-8035-7293 \\
Eugene O.Zdorovtsev & 1 & Zenya7777@ mail.ru & (1) 0000-0002-5126-8723 \\
Lyudmila V. Malakova & 1 & mixbox3@ mail.ru & (1) 0000-0003-0481-4175 \\
Anastasia P. Fursova & 1 & nasti999@ gmail.com & (1) 0000-0003-0481-4290 \\
Alexander E. Stolyarov & 1 & stolyarov1997@ bk.ru & (1) 0000-0003-3086-3280 \\
Ekaterina S. Taltynova & 1 & ekaterynageeva@ mail.ru & (D) 0000-0001-6749-8227x \\
\hline
\end{tabular}

1 Voronezh State University of Engineering Technologies, Revolution Av., 19 Voronezh, 394036, Russia

Abstract. The positive effect on the human body of polyunsaturated fatty acids is confirmed by numerous studies. Therefore, it seems relevant to create food systems for the enrichment of consumer goods with physiologically active and essential substances, in particular polyunsaturated fatty acids. The effect of alimentary shunting of the human body with polyunsaturated fatty acids depends on many factors, the most important are their amount in the diet, composition, ratio of polyunsaturated and saturated fatty acids, the presence of antioxidants.

\section{Для цитирования}

Алексеева Т.В., Здоровцев Е.О., Малакова Л.В., Фурсова А.П., Столяров А.Е., Талтынова Е.С. Моделирование пищевых систем для алиментарной коррекции соотношения полиненасыщенных жирных кислот в организме человека // Вестник ВГУИТ. 2020. T. 82. № 1. С. 70-75. doi:10.20914/2310-1202-2020-1-70-75

\section{(C) 2020, Алексеева Т.В. и др. / Alekseeva T.V. et al.}

\section{For citation}

Alekseeva T.V., Zdorovtsev E.O., Malakova L.V., Fursova A.P., Stolyarov A.E., Taltynova E.S. Modeling of food systems for nutritional correction of the ratio of polyunsaturated fatty acids in people. Vestnik VGUIT [Proceedings of VSUET]. 2020. vol. 82. no. 1. pp. 70-75. (in Russian). doi:10.20914/2310-1202-2020-1-70-75

This is an open access article distributed under the terms of the Creative Commons Attribution 4.0 International License 
The aim of the research is to develop a methodology for managing the quality of food systems to balance the ratio of $\omega-6$ and $\omega-3$ polyunsaturated fatty acids. A software product was developed that allows you to select the ratio of the components in the food composition for the given ratios $\omega-6 / \omega-3$. As an example, a food enrichment system was selected, consisting of oilcake of wheat germ, amaranth and pumpkin oils. The created software product allows you to work with other food systems. As a result of data processing, a nomogram was obtained to determine the percentage ratio of $\omega-6 / \omega-3$ fatty acid components in the food system. A software product has been developed that makes it possible to select the content of the third component in food compositions according to two fixed quantitative values of the components that satisfies the given ratio of $\omega-6 / \omega-3$ acids. A variant of the food composition is proposed and the work of the developed software product is tested. It has been established that a food enrichment system based on cake of wheat germ oil, amaranth oil and pumpkin oils can be used to enrich food products with polyunsaturated fatty acids, and this creates the possibility of targeted regulation of the ratio of $\omega-6 / \omega-3$ fatty acids. The developed software allows, in production conditions, depending on the availability of certain types of raw materials, to select the best options for food compositions and choose the most suitable option in the current production situation, make decisions on the movement of raw materials at the enterprise after analyzing the data.

Keywords: polyunsaturated fatty acids, PUFA balance, omega-3, omega-6, optimization, programming

\section{Введение}

Положительное влияние на организм человека полиненасыщенных жирных кислот (ПНЖК) подтверждено многочисленными исследованиями. Эффект алиментарного шунтирования организма человека ПНЖК зависит от множества факторов, самыми существенными считаются количество ПНЖК в рационе, их состав, соотношение ПНЖК и насыщенных жирных кислот, наличие антиоксидантов. В последнее время значительный интерес отводится наличию в рационах питания у различных групп населения $\omega-6$ и $\omega-3$ полиненасыщенных жирных кислот [1-11].

Согласно нормам физиологических потребностей введен рекомендуемый уровень адекватного потребления $\omega-6$ и $\omega-3$ жирных кислот для взрослых, составляющий соответственно 5-10 и 0,8-1,5 г/сутки при соотношении $\omega-6 / \omega-3$ 5-10:1 [12]. Поэтому представляется весьма актуальным создание пищевых систем для обогащения продуктов массового потребления физиологически активными и эссенциальными веществами, в частности ПНЖК, что согласуется с основами государственной политики РФ в секторе здорового питания населения и «Комплексной программой развития биотехнологий в РФ на период 2020 года» [13].

Цель работы - разработка методики управления качеством пищевых систем для балансирования соотношения $\omega-6$ и $\omega-3$ полиненасыщенных жирных кислот.

\section{Материалы и методы}

В работе использовался метод объектноориентированного программирования на языке Python 2.6, применялась система для статистического анализа данных Statistica 6.1.478. Программный продукт на императивном, структурированном, объектно-ориентированном языке программирования Delphi 7.0. Библиотека математических вычислений AlgLib.

\section{Результаты и обсуждение}

Был разработан программный продукт, позволяющий подбирать соотношения компонентов в пищевой композиции для заданных соотношений $\omega-6 / \omega-3$. В основу оптимизации были заложены рекомендации НИИ питания РАМН.

Для примера была выбрана пищевая обогащающая система (ПОС). Созданный программный продукт позволяет работать и с другими пищевыми системами. Основным компонентом ПОС является жмых зародышей пшеницы (ЖЗП). В жмыхе остаточное количество масла составляет $8 \%$, при этом соотношение $\omega-6: \omega-3$ находится на уровне $3: 1$, что не составляет рекомендуемым нормам $[14,15]$.

Для оптимизации соотношения $\omega-6 / \omega-3$ в ПОС предлагается внести купаж растительных масел семян амаранта и тыквы. Анализ экспериментально определенного жирнокислотного состава масла зародышей пшеницы показал, что в нем незаменимых ПНЖК содержится около $58 \%$ от их общего количества. При содержании масла в ЖЗП 8\% на долю $\omega-6$ и $\omega-3$ приходится 3,4 и 1,2 г в 100 г продукта соответственно.

То есть содержание $\omega-6$ кислот существенно ниже рекомендуемого. Анализируя жирнокислотный состав амарантового и тыквенного масел, можно сделать заключение о том, что ни одно из них не удовлетворяет необходимому соотношению $\omega-6: \omega-3$ и составляет 50:1 и 3:1 соответственно.

В результате обработки данных на разработанном программном продукте была получена номограмма для определения процентного соотношения амарантового, тыквенного масла и ЖЗП, при котором обеспечивается требуемое соотношение $\omega-6 / \omega-3$ жирных кислот. Для требуемого сочетания кислот необходимо выбирать точку на номограмме внутри некоторой области. Программное обеспечение позволяет посчитать под двум зафиксированным измерениям значение третьего, удовлетворяющее заданному 
соотношению. Программный продукт написан на императивном, структурированном, объектноориентированном языке программирования Delphi, который является диалектом Object Pascal. Данный язык идеально подходит для анализа и визуализации данных [14-18].
На рисунках 1-3 представлены варианты скриншотов интерфейсов работающей программы подбора компонентного состава ПОС, которая позволяет находить конкретное количество каждого ингредиента ПОС при заданном соотношении $\omega-6$ и $\omega-3$ жирных кислот.

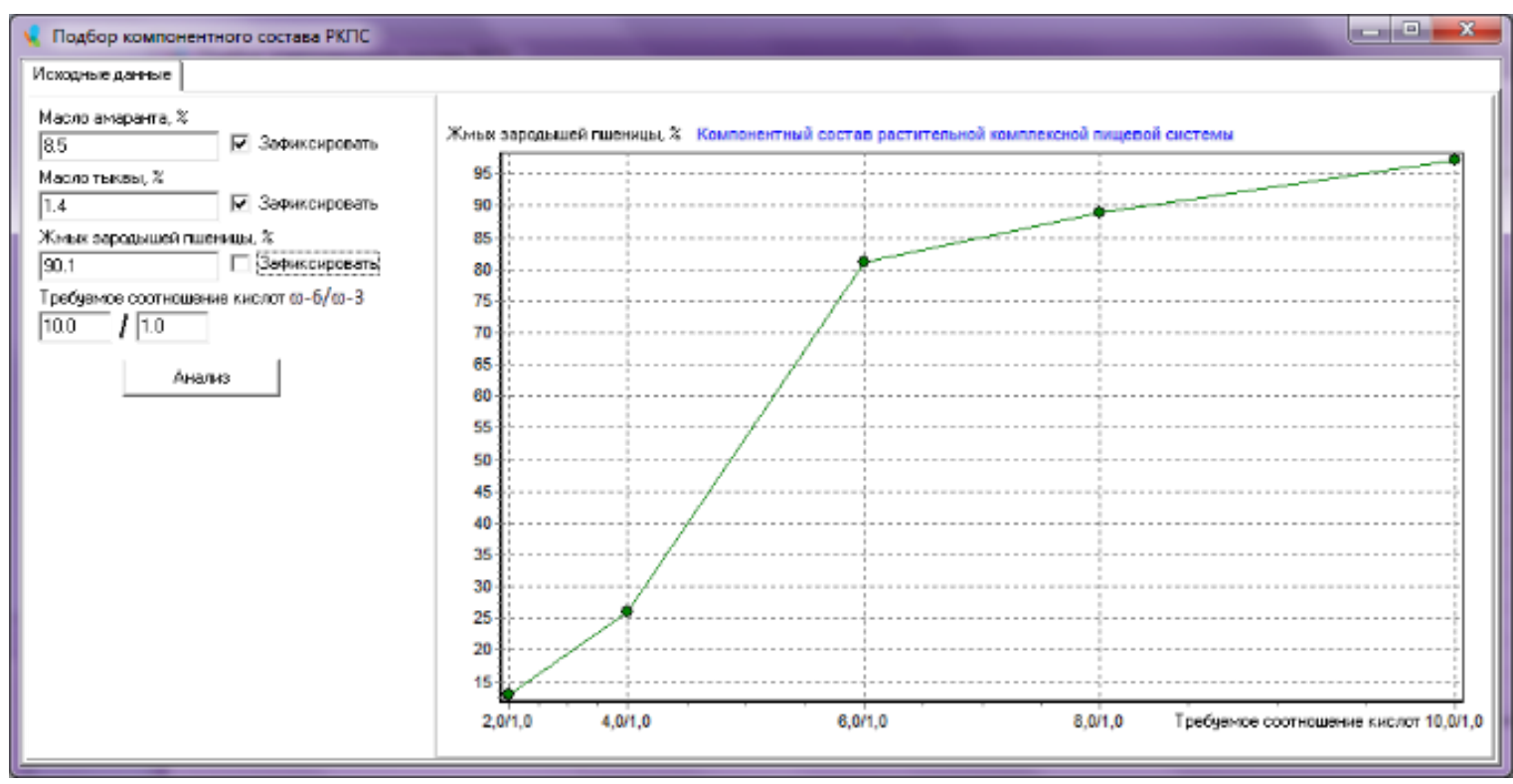

Рисунок 1. Интерфейс программы подбора компонентного состава пищевой обогащающей системы с фиксированными значениями содержания масел амаранта $(8,50 \%)$ и тыквы $(1,40 \%)$ и рассчитанным значением содержания жмыха зародышей пшеницы $(90,10 \%)$ для заданного соотношения кислот $\omega-6 / \omega-3(10,0 / 1,0)$

Figure 1. The interface of the program for selecting the component composition of a food enrichment system with fixed values for the content of amaranth oils $(8,50 \%)$ and pumpkins $(1,40 \%)$ and the calculated value of the content of cake of wheat germ $(90,10 \%)$ for a given ratio of acids $\omega-6 / \omega-3(10,0 / 1,0)$

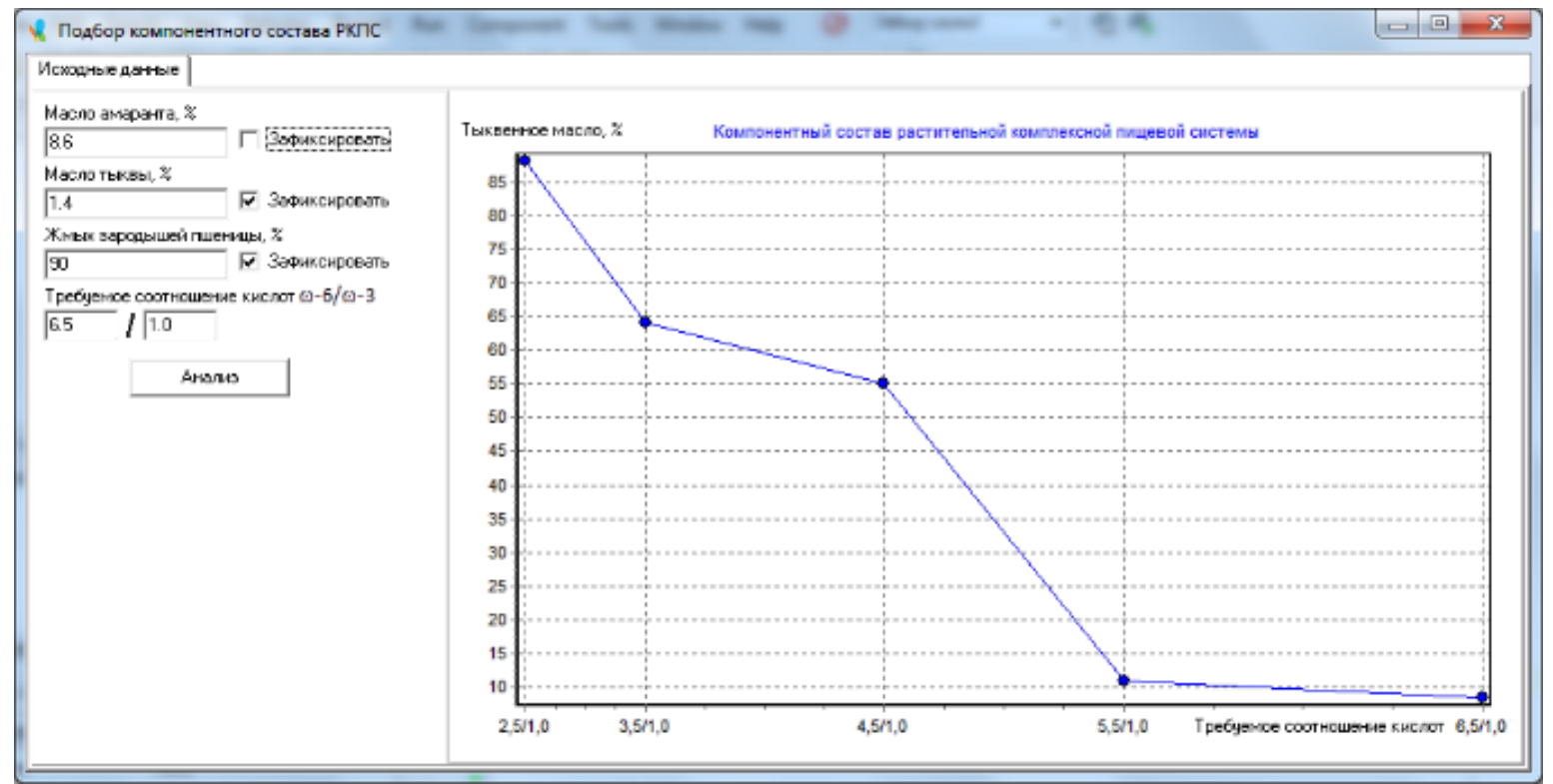

Рисунок 2. Интерфейс программы подбора компонентного состава пищевой обогащающей системы с фиксированными значениями содержания масла тыквы $(1,40 \%)$ и жмыха зародышей пшеницы (90,0\%) и рассчитанным значением масла амаранта $(8,60 \%)$ для заданного соотношения кислот $\omega-6 / \omega-3(6,5 / 1,0)$

Figure 2. The interface of the program for selecting the component composition of a food enrichment system with fixed values of pumpkin oil content $(1,40 \%)$ and wheat germ cake $(90,0 \%)$ and the calculated value of amaranth oil $(8,60 \%)$ for a given ratio of acids $\omega-6 / \omega-3(6,5 / 1,0)$ 


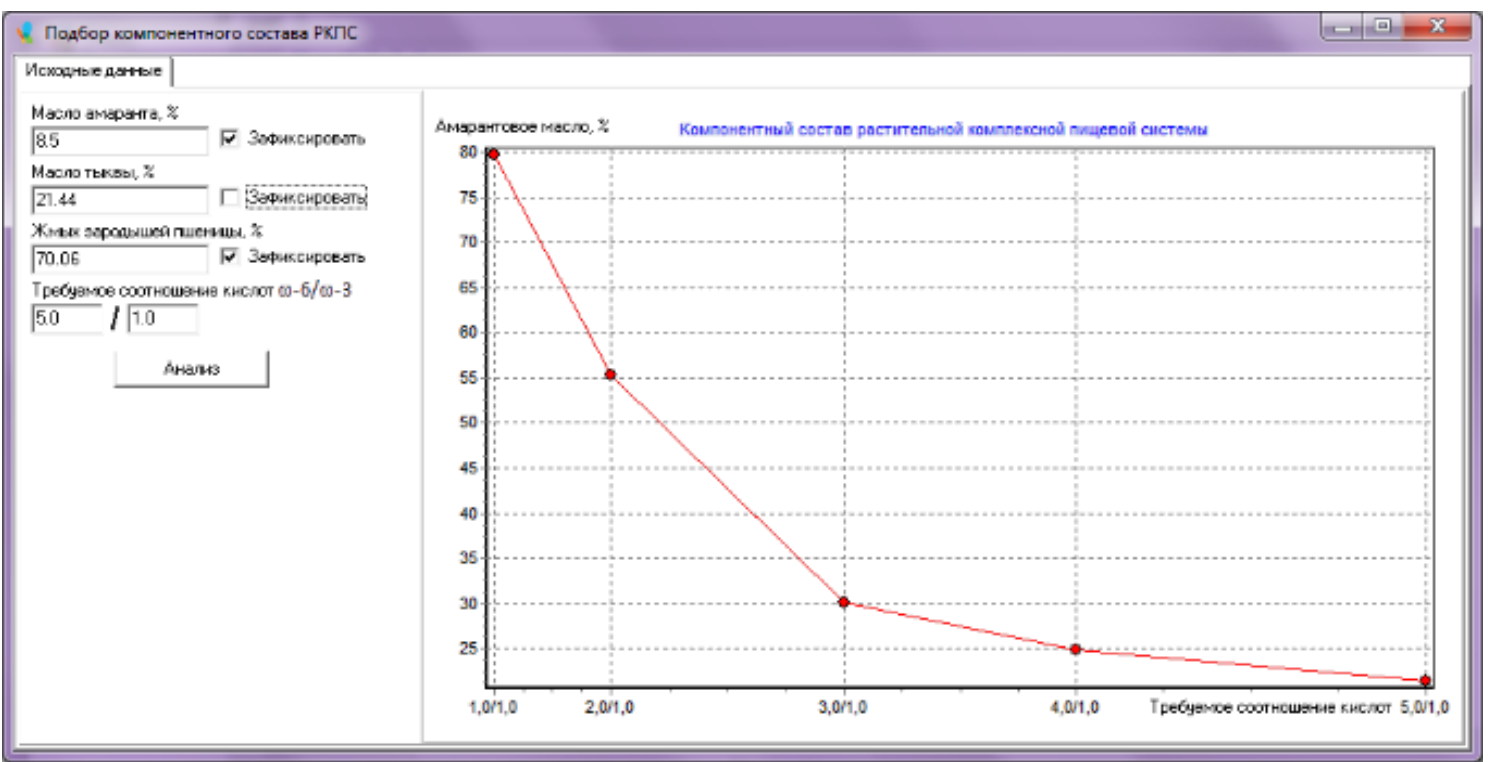

Рисунок 3. Интерфейс программы подбора компонентного состава пищевой обогащающей системы с фиксированными значениями содержания масла амаранта $(8,50 \%)$ и жмыха зародышей пшеницы $(70,06 \%)$ и рассчитанным значением масла тыквы $(21,44 \%)$ для заданного соотношения кислот $\omega-6 / \omega-3(5,0 / 1,0)$

Figure 3. The interface of the program for selecting the component composition of a food enrichment system with fixed values of amaranth oil content $(8,50 \%)$ and wheat germ cake $(70,06 \%)$ and the calculated value of pumpkin oil $(21,44 \%)$ for a given ratio of acids $\omega-6 / \omega-3(5,0 / 1,0)$

На рисунках 1-3 в качестве исходных данных выступают поля для ввода количества тыквенного, амарантового масла и жмыха зародышей пшеницы, а также требуемое соотношение кислот. Из рисунка 1 видно, что напротив полей с содержанием тыквенного и амарантового масла поставлены флаги «Зафиксировать». Это означает, что в дальнейшем в процессе оптимизации эти значения изменяться не будут. После нажатия на кнопку «Анализ» будет произведена оптимизация, результаты которой видны на графике справа. В результате проведенной оптимизации в поле с содержанием ЖЗП появится конкретное значение (в данном случае 90,1\%), а в поле «Требуемое соотношение $\omega-6 / \omega-3$ жирных кислот» для данного случая (ЖЗП - 90,1\%; масло амаранта - 8,5\%; масло тыквы - 1,4\%) будет указано соотношение жирных кислот (в данном примере $\omega-6 / \omega-3$ равно 10:1).

Из рисунка 2 следует, что в этом случае изначально заданы значения содержания масла тыквы $(1,40 \%)$ и ЖЗП $(90,0 \%)$. Результатом оптимизации работы программы является то, что при соотношении $\omega-6 / \omega-3(6,5 / 1,0)$ количество масла амаранта в пищевой композиции будет составлять $8,60 \%$.

Разработанная программа, представленная на рисунке 3 , отличается тем, что в данном случае неизменными являются содержание масла амаранта (8,50\%) и ЖЗП (70,06\%). А в процессе работы программы заданными соотношениями $\omega-6 / \omega-3$ считаются $5,0 / 1,0)$.
При этом рассчитанное количество масла тыквы в смеси равно 21,44\%. Важно отметить, что применение данного программного обеспечения может быть актуальным на промышленных предприятиях для предварительного расчета и варьирования рецептур пищевых смесей с учетом наличия в данный момент конкретных видов сырья с определенными качественными показателями и массой.

\section{Заключение}

Таким образом, при проведении исследований были получены следующие результаты:

- разработан программный продукт, позволяющий подобрать в пищевых композициях по двум зафиксированным количественным значениям компонентов содержание третьего компонента, удовлетворяющего заданному соотношению $\omega-6 / \omega-3$ кислот;

- предложен вариант пищевой композиции и проведена апробация работы разработанного программного продукта;

- установлено, что пищевая обогащающая система на основе жмыха зародышей пшеницы, масел амаранта и тыквы может применяться для обогащения продуктов питания ПНЖК, при этом создается возможность целенаправленного регулирования соотношения $\omega-6 / \omega-3$ жирных кислот.

Разработанное программное обеспечение позволяет в условиях производства в зависимости от наличия определенных наименований сырьевых источников подбирать оптимальные 
варианты пищевых композиций с заведомо известным соотношением $\omega-6 / \omega-3$ и выбирать наиболее приемлемый в сложившейся производственной ситуации вариант, принимать решения по движению сырья на предприятии после анализа полученных данных. Данная программа работает для получения численных значений рассмотренной выше пищевой композиции. Для систем с другим компонентным составом необходимы дополнительные сведения, но программа может быть легко модифицирована при их наличии.

\section{Литература}

1 Антипова Л.В., Родионова Н.С., Попов Е.С. Тенденции развития научных основ проектирования пищевых продуктов // Известия высших учебных заведений. Пищевая технология. 2018. № 1. С. 8-11.

2 Нечаев А.П. Научные основы технологий получения функциональных жировых продуктов нового поколения // Масла и жиры. 2010. № 8. С. 26-27.

3 Степанычева Н.В., Фудько А.А. Купажированные растительные масла с оптимизированным жирнокислотным составом // Химия растительного сырья. 2011. № 2. С. 27-33.

4 Campos H., Baylin A., Willett W. Linolenic Acid and Risk of Nonfatal Acute Myocardial Infarction // Circulation. 2010. V. 118. P. 339-345.

5 Chapkin R., Murray D., Davidson L. Bioactive dietary long chain fatty acids: Emerging mechanisms of action // Br. Journ. Nutr. 2011. V. 100. № 6. P. 1152-1157.

6 Fetterman J., Zdanowicz M. Therapeutic potential of $\omega-3$ polyunsaturated fatty acids in disease // Am. Journ. Health Syst. Pharm. 2011. V. 66. № 13. P. 1169-1179.

7 Jicha G.A., Markesbery W. Omega-3 fatty acids: potential role in the management of early Alzheimer's disease // Clin. Interv. Aging. 2010. V. 5. P. 45-61.

8 Kapoor R., Huang Y. Gamma linolenic acid: an antimflammatory omega-6 fatty acid // Curr. Pharm. Biotechnol. 2011. V. 7. № 6. P. 531-534.

9 Weaver K., Ivester P., Seeds M. Effect of Dietary Fatty Acids on Inflammatory Gene Expression in Healthy Humans // Journ. Biol. Chem. 2009. V. 284. № 23. P. 15400-15407.

10 Winnik S., Lohmann C., Richter E. Dietary a-linolenic acid diminishes experimental atherogenesis and restricts $\mathrm{T}$ cell-driven inflammation // Eur Heart Journ. 2011. P. 53-67.

11 Myhrstad M., Retterstol K., Telle-Hansen V. Effect of marine $\omega-3$ fatty acids on circulating inflammatory markers in healthy subjects and subjects with cardiovascular risk factors // InflammRes. 2011. V. 60. № 34. P. 309-319.

12 Методические рекомендации 2.3.1.2432-08. Нормы физиологических потребностей в энергии и пищевых веществах для различных групп населения РФ. Москва: Госсанэпиднадзор РФ, 2008. 41 с.

13 Основы государственной политики Российской Федерации в области здорового питания населения на период до 2020 года: распоряжение Правительства Российской Федерации от 25 октября 2010 г. № 1873-p. URL: http://www. http://www.rg.ru

14 Алексеева Т.В., Агаева Н.Ю., Калгина Ю.О. Конструирование компонентного состава пищевой композиции для балансирования ПНЖК - состава // Теория и практика персонализированного питания. 2019. № 2. С. 75-85.

15 Родионова Н.С., Алексеева Т.В. Технология пищевых продуктов сбалансированного ПНЖК состава: монография. Воронеж: ВГУИТ, 2015. 257 с.

16 Кандзюба С.П., Громов В.Н. Delphi 6/7. Базы данных и приложения. СПб: ООО «ДиаСофт», 2012.340 с.

17 Роб П., Коронел К. Системы баз данных: проектирование, реализация и управление. Санкт-Петербург: BHV, 2010.450 с.

18 Belokurov S.V., Rodionova N.S., Belokurova E.V., Alexeeva T.V. Modeling of process of lifting power change of baker's yeast pressed depending on nature and quantity of introduced vegetable component // Journal of Physics: International Conference Information Technologies in Business and Industry. 2018. P. 1-4.

\section{References}

1 Antipova L.V., Rodionova N.S., Popov E.S. Trends in the development of the scientific basis for food design. News of higher educational institutions. Food technology. 2018. no. 1. pp. 8-11. (in Russian).

2 Nechaev A.P. The scientific basis of the technology for the production of functional fat products of a new generation. Oils and Fats. 2010. no. 8. pp. 26-27. (in Russian).

3 Stepanycheva N.V., Fudko A.A. Blended vegetable oils with optimized fatty acid composition. Chemistry of plant raw materials. 2011. no. 2. pp. 27-33. (in Russian).

4 Campos H., Baylin A., Willett W. Linolenic Acid and Risk of Nonfatal Acute Myocardial Infarction. Circulation. 2010. vol. 118. pp. 339-345.

5 Chapkin R., Murray D., Davidson L. Bioactive dietary long chain fatty acids: Emerging mechanisms of action. Br. Journ. Nutr. 2011. vol. 100. no. 6. pp. 1152-1157.

6 Fetterman J., Zdanowicz M. Therapeutic potential of $\omega-3$ polyunsaturated fatty acids in disease. Am. Journ. Health Syst. Pharm. 2011. vol. 66. no. 13. pp. 1169-1179.

7 Jicha G.A., Markesbery W. Omega-3 fatty acids: potential role in the management of early Alzheimer's disease. Clin. Interv. Aging. 2010. vol. 5. pp. 45-61.

8 Kapoor R., Huang Y. Gamma linolenic acid: an antimflammatory omega-6 fatty acid. Curr. Pharm. Biotechnol. 2011. vol. 7. no. 6. pp. 531-534.

9 Weaver K., Ivester P., Seeds M. Effect of Dietary Fatty Acids on Inflammatory Gene Expression in Healthy Humans. Journ. Biol. Chem. 2009. vol. 284. no. 23. pp. 15400-15407.

10 Winnik S., Lohmann C., Richter E. Dietary a-linolenic acid diminishes experimental atherogenesis and restricts $\mathrm{T}$ cell-driven inflammation. Eur Heart Journ. 2011. pp. 53-67.

11 Myhrstad M., Retterstol K., Telle-Hansen V. Effect of marine $\omega$-3 fatty acids on circulating inflammatory markers in healthy subjects and subjects with cardiovascular risk factors. InflammRes. 2011. vol. 60. no. 34. pp. 309-319. 
12 Guidelines 2.3.1.2432-08. Norms of physiological needs for energy and nutrients for various population groups of the Russian Federation. Moscow, State Sanitary Inspection of the Russian Federation, 2008. 41 p. (in Russian).

13 Fundamentals of the state policy of the Russian Federation in the field of healthy nutrition for the period up to 2020: Order of the Government of the Russian Federation dated October 25, 2010. № 1873-r. Available at: http: // www. http://www.rg.ru (in Russian).

14 Alekseeva T.V., Agaeva N.Yu., Kalgina Yu.O. The construction of the component composition of a food composition for balancing PUFA - composition. Theory and practice of personalized nutrition. 2019. no. 2. pp. 75-85. (in Russian).

15 Rodionova N.S., Alekseeva T.V. Food technology balanced PUFA composition: monograph. Voronezh: VSUET, 2015. 257 p. (in Russian).

16 Kandzyuba S.P., Gromov V.N. Delphi 6/7. Databases and applications. St. Petersburg, DiaSoft LLC, 2012. 340 p. (in Russian).

17 Rob P., Coronel K. Database systems: design, implementation and management. St. Petersburg, BHV, 2010. 450 p. (in Russian).

18 Belokurov S.V., Rodionova N.S., Belokurova E.V., Alexeeva T.V. Modeling of process of lifting power change of baker's yeast pressed depending on nature and quantity of introduced vegetable component. Journal of Physics: International Conference Information Technologies in Business and Industry. 2018. pp. 1-4.

\section{Сведения об авторах}

Татьяна В. Алексеева д.т.н., профессор, кафедра сервиса и ресторанного бизнеса, пр-т Революции, 19, Воронеж, 394036 , Россия, пр-т Революции, 19, Воронеж, 394036, Россия, zyablova@mail.ru

(Dhttps://orcid.org/0000-0001-8035-7293

Евгений О. Здоровцев магистр, кафедра сервиса и ресторанного бизнеса, Воронежский государственный университет инженерных технологий, пр-т Революции, 19, Воронеж, 394036, Россия, Zenya7777@ mail.ru

(Dhttps://orcid.org/0000-0002-5126-8723

Людмила В. Малакова магистр, кафедра сервиса и ресторанного бизнеса, Воронежский государственный университет инженерных технологий, пр-т Революции, 19, Воронеж, 394036, Россия, mixbox3@mail.ru

(Dhttps://orcid.org/0000-0003-0481-4175

Анастасия П. Фурсова магистр, кафедра сервиса и ресторанного бизнеса, Воронежский государственный университет инженерных технологий, пр-т Революции, 19, Воронеж, 394036, Россия, nasti999@ gmail.com

(Dhttps://orcid.org/0000-0003-0481-4290

Александр Е. Столяров магистр, кафедра сервиса и ресторанного бизнеса, Воронежский государственный университет инженерных технологий, пр-т Революции, 19, Воронеж, 394036, Россия, stolyarov1997@bk.ru

(Dhttps://orcid.org/0000-0003-3086-3280

Екатерина С. Талтынова магистр, кафедра сервиса и ресторанного бизнеса, Воронежский государственный университет инженерных технологий, пр-т Революции, 19, Воронеж, 394036, Россия, ekaterynageeva@mail.ru

(Dhttps://orcid.org/0000-0001-6749-8227x

\section{Вклад авторов}

Татьяна В. Алексеева написала рукопись, корректировала её до подачи в редакцию и несет ответственность за плагиат

Евгений О. Здоровцев обзор литературных источников по исследуемой проблеме, провела эксперимент

Людмила В. Малакова корректировка рукописи до подачи в редакцию

Анастасия П. Фурсова обзор литературных источников по исследуемой проблеме, выполнила расчёты

Александр Е. Столяров предложил методику проведения эксперимента

Екатерина С. Талтынова выполнила расчеты

Конфликт интересов

Авторы заявляют об отсутствии конфликта интересов.

\section{Information about authors}

Tatyana V. Alekseeva Dr. Sci. (Engin.), professor, service and restaurant business department, Voronezh State University of Engineering Technologies, Revolution Av., 19 Voronezh, 394036, Russia, zyablova@mail.ru

Dhttps://orcid.org/0000-0001-8035-7293

Eugene O. Zdorovtsev master student, service and restaurant business department, Voronezh State University of Engineering Technologies, Revolution Av., 19 Voronezh, 394036, Russia, Zenya7777@mail.ru

(Dhttps://orcid.org/0000-0002-5126-8723

Lyudmila V. Malakova master student, service and restaurant business department, Voronezh State University of Engineering Technologies, Revolution Av., 19 Voronezh, 394036, Russia, mixbox3@mail.ru

(D)https://orcid.org/0000-0003-0481-4175

Anastasia P. Fursova master student, service and restaurant business department, Voronezh State University of Engineering Technologies, Revolution Av., 19 Voronezh, 394036, Russia, nasti999@gmail.com

Dhttps://orcid.org/0000-0003-0481-4290

Alexander E. Stolyarov master student, service and restaurant business department, Voronezh State University of Engineering Technologies, Revolution Av., 19 Voronezh, 394036, Russia, stolyarov1997@bk.ru

Dhttps://orcid.org/0000-0003-3086-3280

Ekaterina S. Taltynova master student, service and restaurant business department, Voronezh State University of Engineering Technologies, Revolution Av., 19 Voronezh, 394036, Russia, ekaterynageeva@mail.ru

Dhttps://orcid.org/0000-0001-6749-8227x

\section{Contribution}

Tatyana V. Alekseeva All authors are equally involved in the writing of the manuscript and are responsible for plagiarism

Eugene O. Zdorovtsev review of literary sources on the investigated problem, conducted an experiment

Lyudmila V. Malakova correction of the manuscript before submission to the editor

Anastasia P. Fursova review of the literature on an investigated problem, conducted an experiment, performed computations

Alexander E. Stolyarov proposed a scheme of the experiment and organized production trials

Ekaterina S. Taltynova performed calculations

\section{Conflict of interest}

The authors declare no conflict of interest.

\begin{tabular}{ccc}
\hline Поступила 03/02/2020 & После редакции 15/02/2020 & Принята в печать 24/02/2020 \\
\hline Received 03/02/2020 & Accepted in revised 15/02/2020 \\
\hline
\end{tabular}

